\title{
An Extended Exponentiated Exponential Distribution and its Properties
}

\author{
S. E Abu-Youssef \\ Department of Mathematics, \\ Faculty of Science, \\ Al-Azhar University, \\ Egypt
}

\author{
B. I Mohammed \\ Department of Mathematics, \\ Faculty of Science, \\ Al-Azhar University, \\ Egypt
}

\author{
M. G Sief \\ Department of Mathematics, \\ Faculty of Science, \\ Fayoum University, \\ Egypt
}

\begin{abstract}
In this paper, we introduce an extension of the exponentiated exponential $(E E)$ distribution which offers a more flexible model for lifetime data. This model is generated by compound distribution with mixing exponential model. Several statistical and reliability properties of the proposed distribution are explored as the geometric extreme stability, sufficient conditions for the shape behavior of the density and hazard rate functions, the moments and mean residual life time. Estimation of unknown parameters using the maximum likelihood are obtained. Moreover, an application to a real data set is presented for illustrative purposes.
\end{abstract}

\section{Keywords}

Exponentiated Exponential Distribution, Compound distribution, Geometric extreme stability, AIC, BIC, Likelihood ratio test, P-P plot, mean residual life

\section{INTRODUCTION}

The two parameter $E E$ distribution introduced by Ahuja and Nash [2] and further, studied by Gupta and Kundu[6]. This distribution has shape and scale parameters like Gamma and Weibull distributions. Moreover it has better fit than Weibull and Gamma in many cases, Raja and $\operatorname{Mir}[15]$. The applications of the $E E$ distribution have been widespread, we mention: models to determine bout criteria for analysis of animal behavior, Yeates et al. [21]; software reliability growth models for vital quality metrics, Subburaj et al. [11]; models for episode peak and duration for eco-hydro-climatic applications, Biondi et al. [5]; and cure rate modeling, Kannan et al. [8]. An absolutely continuous random variable $(r v)$ is said to have The $E E$ distribution if its survival function $(s f)$ and its probability density function $(p d f)$ are given by,

$$
\begin{gathered}
\bar{F}(x)=1-\left(1-e^{-\beta x}\right)^{\alpha}, \\
f(x)=\alpha \beta e^{-\beta x}\left(1-e^{-\beta x}\right)^{\alpha-1}
\end{gathered}
$$

respectively, for $x>0, \alpha>0$ and $\beta>0$. We write $X \sim$ $E E(\alpha, \beta)$, here $\alpha$ is the shape parameter and $\beta$ is the scale parameter. In the particular case $\alpha=1$, it represents the exponential family. therefore, all three families, Gamma, Weibull and the $E E$ are generalization of the exponential family but in different ways.
Many authors derived other properties of the $(E E)$ distribution, Gupta and Kundu [7], Raqab [16], Zheng [22], Shirke et al. [18],9 Abdel-Hamid and Al-Hussaini [1], Kundu and Pradhan [10] and Aslam et al. [4]. Some generalizations of the $E E$ distribution are discussed in Nadarajah and Kotz [13]. On the other hand, MarshallOlkin [12] defined a technique of generalization for a given probability distribution increasing the number of parameters by one. The new parameter results a flexibility in the distribution and known as the Marshall-Olkin extended distribution.

Assume that $X$ is a rv with a given a survival function $(s f) \bar{F}(x)$, the Marshall-Olkin extension of the original family is defined to be the family of distributions with $s f$ as:

$$
\bar{G}(x)=\frac{\lambda \bar{F}(x)}{1-\bar{\lambda} \bar{F}(x)}, \quad-\infty<x<\infty, \lambda>0, \bar{\lambda}=1-\lambda .
$$

In this article, we introduce a new variant of the Marshall-Olkin extended family of distributions by selecting in 3 the $E E$ distribution with $s f[1$ which yields

$$
\bar{G}(x)=\frac{\lambda-\lambda\left(1-e^{-\beta x}\right)^{\alpha}}{\lambda+\bar{\lambda}\left(1-e^{-\beta x}\right)^{\alpha}}, \quad x>0, \alpha>0, \lambda>0, \beta>0 .
$$

We refer to the distribution with $s f$ given by 4 as Marshall-Olkin extended $E E$ distribution with parameters $(\alpha, \beta, \lambda)$ and will be denoted by $M O E E E$ distribution. The aim of this article is to reveal some statistical properties of the MOEEE distribution. These properties include: $(i)$ expressing this proposed distribution as a compounding process with exponential mixing model; $(i i)$ shapes of the density function; $(i i i)$ moments and quantiles; $(i v)$ shapes of the hazard rate function; finally, $(v)$ utilizing maximum likelihood estimation, the proposed distribution is fitted to random real data.

\section{COMPOUNDING}

Let $\bar{G}(x \mid \theta)$ be the conditional $s f$ of a continuous $r v X$ given a continuous $r v \Theta$. Let $\Theta$ follows a distribution with the $p d f m(\theta)$. A distribution with the $s f$

$$
\bar{G}(x)=\int_{-\infty}^{\infty} \bar{G}(x \mid \theta) m(\theta) d \theta, \quad-\infty<x<\infty
$$

is called a compound distribution with mixing density $m(\theta)$. Compound distributions provide a useful tool to get new class of distributions in terms of existing ones. The following theorem shows that 
the $M O E E E$ distribution can be expressed by the compounding distribution.

THEOREM 1. Suppose that the conditional sf of a continuous $r v X$ given a continuous $r v \Theta=\theta$ is given by:

$$
\bar{G}(x \mid \theta)=\exp \left(\theta\left(1-\frac{1}{1-\left(1-e^{-\beta x}\right)^{\alpha}}\right)\right)
$$

and let $\Theta$ have an exponential distribution with the pdf,

$$
m(\theta)=\theta e^{-\lambda \theta}
$$

then the compound distribution of $X$ is the $\operatorname{MOEEE}(\alpha, \beta, \lambda)$.

Proof. For all $x>0, \beta, \theta, \lambda>0$, the unconditional $s f$ of $X$ is given by

$$
\begin{aligned}
\bar{G}(x) & =\int_{-\infty}^{\infty} \bar{G}(x \mid \theta) m(\theta) d \theta \\
& =\lambda \int_{0}^{\infty} \exp \left(-\theta\left(\frac{1}{1-\left(1-e^{-\beta x}\right)^{\alpha}}-\bar{\lambda}\right) d \theta\right. \\
& =\frac{\lambda-\lambda\left(1-e^{-\beta x}\right)^{\alpha}}{\lambda+\bar{\lambda}\left(1-e^{-\beta x}\right)^{\alpha}}
\end{aligned}
$$

which is the $s f$ of the $M O E E E(\alpha, \beta, \lambda)$ distribution.

In the next section we will show that the MOEEE distribution is geometric extreme stable.

\section{GEOMETRIC EXTREME STABLE}

For independent identical distributed (iid) $r v$ 's $X_{1}, X_{2}, \ldots, X_{N}$ with $s f(4)$ and $N$ is independent of the $X_{i}^{\prime}$ s with geometric $(p)$ distribution, then $U_{N}=\min \left(X_{1}, X_{2}, \ldots, X_{N}\right), V_{N}=$ $\max \left(X_{1}, X_{2}, \ldots, X_{N}\right)$, have the distribution in the same family with $0<\alpha=p \leq 1$ and $\alpha=1 / p \geq 1$ respectively. The following theorem shows that $M O E E E$ distribution geometric minimum and maximum stable.

THEOREM 2. The MOEEE distribution is geometric extreme stable

Proof. let $X_{1}, X_{2}, \ldots, X_{N}$ be a sequence of iid $r v^{\prime}$ s. Suppose $N$ is independent of the $X_{i}^{\prime}$ s with geometric distribution :

$$
P_{r}(N=n)=(1-p)^{n-1} p, n=1,2, \ldots
$$

and let

$$
U_{N}=\min \left(X_{1}, X_{2}, \ldots, X_{N}\right), V_{N}=\max \left(X_{1}, X_{2}, \ldots, X_{N}\right),
$$

then

$$
\begin{array}{rlr}
\bar{G}(x) & =P_{r}\left(U_{N}>x\right) \\
& =P_{r}\left(X_{1}>x, X_{2}>x, \ldots, X_{N}>x\right) \\
& =\sum_{n=1}^{\infty} \bar{F}^{n}(x)(1-p)^{n-1} p & \\
& =\frac{p \bar{F}(x)}{1-(1-p) \bar{F}(x)}, \quad-\infty<x<\infty
\end{array}
$$

and

$$
\begin{aligned}
H(x) & =P_{r}\left(V_{N}<x\right) \\
& =P_{r}\left(X_{1}<x, X_{2}<x, \ldots, X_{N}<x\right) \\
& =\sum_{n=1}^{\infty} \bar{F}^{n}(x)(1-p)^{n-1} p \\
& =\frac{p F(x)}{1-(1-p) F(x)}
\end{aligned}
$$

so that

$$
\bar{H}(x)=\frac{\bar{F}(x)}{1-(1-p) \bar{F}(x)} .
$$

Now suppose that

$$
\bar{F}(x)=\frac{\lambda-\lambda\left(1-e^{-\beta x}\right)^{\alpha}}{\lambda+\bar{\lambda}\left(1-e^{-\beta x}\right)^{\alpha}},
$$

which is thesf of the MOEEE, then

$$
\bar{G}(x)=\frac{p \lambda\left(1-\left(1-e^{-\beta x}\right)^{\alpha}\right)}{p \lambda+(1-p \lambda)\left(1-e^{-\beta x}\right)^{\alpha}} .
$$

So $U_{N}$ is geometric minimum stable. Also we have,

$$
\bar{H}(x)=\frac{\lambda / p\left(1-\left(1-e^{-\beta x}\right)^{\alpha}\right)}{\lambda / p+(1-\lambda / p)\left(1-e^{-\beta x}\right)^{\alpha}} .
$$

Hence $V_{N}$ is geometric maximum stable. Therefore the family of $M O E E E$ distribution with $s f(4)$ is geometric extreme stable.

\section{DENSITY, QUANTILES, MOMENTS}

The $p d f$ of the MOEEE with $s f(4)$ is given by

$$
g(x)=\frac{\alpha \beta \lambda\left(1-e^{-\beta x}\right)^{\alpha-1} e^{-\beta x}}{\left(\bar{\lambda}\left(1-e^{-\beta x}\right)^{\alpha}+\lambda\right)^{2}}, \quad x>0 .
$$

The $M O E E E$ distribution having exponential, $E E$ and MarshallOlkin extended exponential ( $M O E E$ ) distributions as sub-models:

(1) If $\alpha=1$, we have the $M O E E$ distribution, Singh et al [19].

(2) If $\lambda=1$ we get the $E E$ distribution, Gupta and Kundu [6].

(3) If $\lambda=1, \alpha=1$ we get the exponential distribution.

The following theorem gives simple conditions under which the $p d f(6)$ is decreasing or unimodal.

THEOREM 3. The pdf of the MOEEE $(\alpha, \beta, \lambda)$ is decreasing (unimodal) if $0<\alpha<1(\alpha>1)$ independent for $\lambda, \beta$.

Proof. The first derivative of $g(x)$ is given by,

$$
g^{\prime}(x)=\frac{\alpha \lambda \beta^{2}\left(1-e^{-\beta x}\right)^{\alpha}}{\left(e^{\beta x}-1\right)^{2}\left(\bar{\lambda}\left(1-e^{-\beta x}\right)^{\alpha}+\lambda\right)^{2}} \quad \phi(x) \quad, x>0
$$

where

$$
\phi(x)=\frac{e^{\beta x}\left(-\bar{\lambda}\left(1-e^{-\beta x}\right)^{\alpha}-\lambda\right)+\alpha\left(-\bar{\lambda}\left(1-e^{-\beta x}\right)^{\alpha}+\lambda\right)}{\left(\bar{\lambda}\left(1-e^{-\beta x}\right)^{\alpha}+\lambda\right)} .
$$

If $\phi(0)=(-\lambda+\lambda \alpha) / \lambda=(\alpha-1) \leq 0$, and $\alpha \in(0,1]$ then $\phi(x) \leq 0$ for all $x>0$, and hence, $g^{\prime}(x) \leq 0$ for all $x>0$ i.e., $\mathrm{g}(\overline{\mathrm{x}})$ is decreasing. For $\alpha>1, \phi(0)=(\alpha-1) \geq 0$, then $\phi(x) \geq 0$ for all $x>0$, and hence, $g^{\prime}(x) \geq 0$ for all $x>0$ i.e., $\mathrm{g}(\mathrm{x})$ is increasing. Since $\lim _{x \rightarrow 0} g(x)=0$ and $\lim _{x \rightarrow \infty} g(x)=0$, 
therefore the $p d f$ of $M O E E E$ first increase and then decreases to zero and hence it has a mode given by,

$$
\alpha(\lambda-1) u^{\alpha+1}-(\lambda-1)(1+\alpha) u^{\alpha}+\alpha \lambda u+\lambda(1-\alpha)=0
$$

where $u=1-e^{-\beta x_{\text {mode }}}$.

Figure 1 below shows the $p d f$ curves for the MOEEE distribution for selected values of the parameters $\alpha, \beta$ and $\lambda$.

If $X$ has $M O E E E$ distribution then the $r$ th moment of $X$ can be
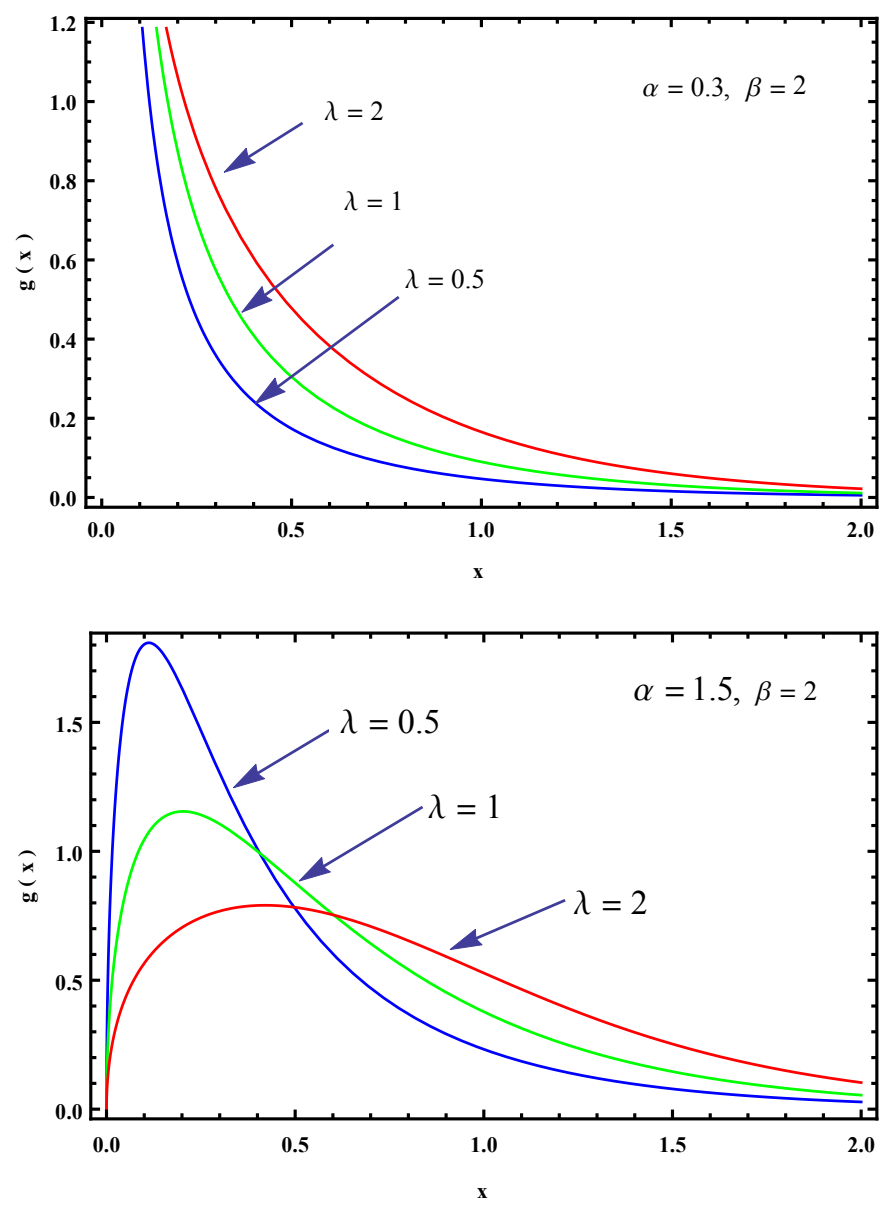

Fig. 1: The $p d f$ of the $M O E E E$ distribution for selected values of the parameters $\lambda, \alpha$ and $\beta$.

written as

$$
\begin{aligned}
E\left(X^{r}\right) & =r \int_{0}^{\infty} x^{r-1} \bar{G}(x) d x \\
& =r \int_{0}^{\infty} x^{r-1} \frac{\lambda-\lambda\left(1-e^{-\beta x}\right)^{\alpha}}{\lambda+\bar{\lambda}\left(1-e^{-\beta x}\right)^{\alpha}} d x \\
& =\frac{r(-1)^{r-1}}{\beta^{r}} \int_{0}^{1}(\ln (1-u))^{r-1} \frac{1-u^{\alpha}}{\left(1-\frac{\lambda-1}{\lambda} u^{\alpha}\right)(1-u)} d u
\end{aligned}
$$

Since $(1-t)^{-1}=\sum_{i=0}^{\infty} t^{i}$ for $|t|<1$, then for $\lambda>1$ we have

$$
\begin{aligned}
E\left(X^{r}\right)= & \frac{r(-1)^{r-1}}{\beta^{r}} \sum_{k=0}^{\infty} \sum_{j=0}^{\infty}\left(\frac{\lambda-1}{\lambda}\right)^{k} \\
& \times \int_{0}^{1} u^{k \alpha+j}\left(1-u^{\alpha}\right)(\ln (1-u))^{r-1} d u \\
= & \frac{r(-1)^{r-1}}{\beta^{r}} \sum_{k=0}^{\infty} \sum_{j=0}^{\infty}\left(\frac{\lambda-1}{\lambda}\right)^{k} \\
& \times \int_{0}^{1}\left(u^{k \alpha+j}-u^{k \alpha+\alpha+j}\right)(\ln (1-u))^{r-1} d u \\
= & \frac{r}{\beta^{r}} \sum_{k=0}^{\infty} \sum_{j=0}^{\infty}\left(\frac{\lambda-1}{\lambda}\right)^{k} \\
& \times \int_{0}^{\infty}\left(\left(1-e^{-y}\right)^{k \alpha+j}-\left(1-e^{-y}\right)^{k \alpha+\alpha+j}\right) y^{r-1} e^{-y} d u
\end{aligned}
$$

using the fact that $\left(1-e^{-y}\right)^{i}=\sum_{m=0}^{\infty} \frac{(-(i))_{m}}{m !}\left(e^{-y}\right)^{m}$ where, $(-(i))_{m}=(-1)^{m}(i-l+1)_{l}$ and $(n)_{l}=n(n+1) \ldots(n+l-1)$ then we get,

$$
\begin{array}{r}
E\left(X^{r}\right)=\frac{1}{\beta^{r}} \sum_{k=0}^{\infty} \sum_{j=0}^{\infty}\left(\frac{\lambda-1}{\lambda}\right)^{k}\left\{\sum_{m=0}^{\infty} \frac{(-(\alpha j+k))_{m}}{m !(m+1)^{r}}\right. \\
\left.-\sum_{l=0}^{\infty} \frac{(-(\alpha j+\alpha+k))_{l}}{l !(l+1)^{r}} \Gamma(r+1)\right\}
\end{array}
$$

Now, the median $X_{\text {median }}$ and the $q^{\text {th }}$ quantile $X_{q}$ of the $M O E E E$ distribution are given respectively by :

$$
G(x)=1-\frac{\lambda-\lambda\left(1-e^{-\beta x}\right)^{\alpha}}{\lambda+\bar{\lambda}\left(1-e^{-\beta x}\right)^{\alpha}}=1 / 2,
$$

then $X_{\text {median }}=1 / \beta \ln \left(\frac{1}{1-\left(\frac{\lambda}{1+\lambda}\right)^{1 / \alpha}}\right)$, to get the $X_{q}$, we have,

$$
G(x)=1-\frac{\lambda-\lambda\left(1-e^{-\beta x}\right)^{\alpha}}{\lambda+\bar{\lambda}\left(1-e^{-\beta x}\right)^{\alpha}}=q,
$$

then $X_{q}=\frac{1}{\beta} \ln \left(\frac{1}{1-\left(\frac{\lambda q}{\lambda q-q+1}\right)^{1 / \alpha}}\right) \quad$ Also, we can obtain the quantiles and the median of the $M O E E, E E$ and the exponential distributions as special cases of that of the MOEEE distribution as follows,

(1) For $\alpha=1$, we have the median and the quantiles of the MOEE distribution as

$X_{\text {median }}=1 / \beta \ln (1+\lambda)$ and $X_{q}=1 / \beta \log \left(1-\frac{\lambda q}{q-1}\right)$, Singh et al[19].

(2) For $\lambda=1$, we have the median and the quantiles of the $E E$ distribution as,

$X_{\text {median }}=1 / \beta \ln \left(\frac{1}{1-\left(\frac{1}{2}\right)^{1 / \alpha}}\right)$ and $X_{q}=1 / \beta \ln \left(\frac{1}{1-q^{1 / \alpha}}\right)$, Gupta and Kundu [6].

(3) For $\alpha=\lambda=1$, we have the median and the quantiles of the exponential distribution as, $X_{\text {median }}=1 / \beta \ln 2$ and $X_{q}=$ $1 / \beta \ln \left(\frac{1}{1-q}\right)$. 


\section{HAZARD RATE FUNCTION AND MEAN RESIDUAL LIFE}

A basic quantity, foundational in survival analysis, is the hazard function. this function is particularly useful in determining the appropriate failure distributions utilizing qualitative information about the mechanism of failure and for describing the way in which the chance of experiencing the event changes with time. The hazard rate function $(h r f)$ of the $M O E E E$ distribution is given by

$$
r(x)=\frac{\alpha \beta\left(1-e^{-\beta x}\right)^{\alpha-1} e^{-\beta x}}{\left[\left(1-e^{-\beta x}\right)^{\alpha}-1\right]\left[(\lambda-1)\left(1-e^{-\beta x}\right)^{\alpha}-\lambda\right]}
$$

Note that for all $\alpha>0$, we have,

$$
r(0)=\left\{\begin{array}{cc}
\infty & 0<\alpha<1, \text { for all } \lambda, \\
\beta & \alpha=1, \lambda=1, \\
0 & \alpha>1, \text { for all } \lambda
\end{array}\right.
$$

and,

$r(\infty)=\beta$ for all $\lambda>0, \alpha>0$

The following theorem describe the behavior of $r(x)$.

THEOREM 4. For any $\beta, \lambda$ the MOEEE distribution has unimodal $h r f$ if $\alpha>1$ and decreasing hrf if $0<\alpha<1$. Also, if $\alpha=1$ then the hrf is decreasing for $\lambda<1$, increasing $\lambda>1$ and constant for $\lambda=1$ for all $x>0$.

PROOF. The proof is similar to that of theorem 3

Figure 2 shows the $h r f$ curves for the MOEEE distribution for selected values of the parameters $\alpha, \beta$ and $\lambda$.

Another ageing property for $M O E E E$ distribution is the mean residual life $(\mathrm{mrl})$, was introduced by Watson and Wells [20] to analyse burn-in problems, has been studied by reliabilists, statisticians, survival analysts and others. Its defined simply as the expected value of the remaining lifetime beyond an age $t$. If $X$ is MOEEE $r v$ with $s f(4)$, The residual life $r v$ at age $t$, denoted by $X_{t}=X-t \mid X>t$. The $m r l$ is defined formally as,

$$
\begin{aligned}
\mu(t)= & E(X-t \mid X>t)=\frac{1}{\bar{G}(t)} \int_{t}^{\infty} \bar{G}(x) d x \\
= & \frac{1+\frac{\bar{\lambda}}{\lambda}\left(1-e^{-\beta t}\right)^{\alpha}}{1-\left(1-e^{-\beta t}\right)^{\alpha}} \int_{t}^{\infty} \frac{1-\left(1-e^{-\beta x}\right)^{\alpha}}{1-\frac{\lambda-1}{\lambda}\left(1-e^{-\beta x}\right)^{\alpha}} d x \\
= & 1 / \beta \frac{1+\frac{\bar{\lambda}}{\lambda}\left(1-e^{-\beta t}\right)^{\alpha}}{1-\left(1-e^{-\beta t}\right)^{\alpha}} \int_{1-e^{-\beta t}}^{1} \frac{1-u^{\alpha}}{\left(1-\frac{\lambda-1}{\lambda} u^{\alpha}\right)(1-u)} d u \\
= & \frac{1+\frac{\bar{\lambda}}{\lambda}\left(1-e^{-\beta t}\right)^{\alpha}}{\beta\left(1-\left(1-e^{-\beta t}\right)^{\alpha}\right)} \sum_{k=0}^{\infty} \sum_{j=0}^{\infty}\left(\frac{\lambda-1}{\lambda}\right)^{k} \\
& \times \int_{1-e^{-\beta t}}^{1} u^{k \alpha+j}\left(1-u^{\alpha}\right) d u \\
= & 1 / \beta \frac{1+\frac{\bar{\lambda}}{\lambda}\left(1-e^{-\beta t}\right)^{\alpha}}{1-\left(1-e^{-\beta t}\right)^{\alpha}} \sum_{k=0}^{\infty} \sum_{j=0}^{\infty}\left(\frac{\lambda-1}{\lambda}\right)^{k} \psi_{k j}(t)
\end{aligned}
$$

where,

$$
\begin{aligned}
\psi_{k j}(t)= & \frac{\alpha}{(k \alpha+j+1)(k \alpha+\alpha+j+1)} \\
& +\frac{\left(1-e^{-\beta t}\right)^{k \alpha+\alpha+j+1}}{k \alpha+\alpha+j+1}-\frac{\left(1-e^{-\beta t}\right)^{k \alpha+j+1}}{k \alpha+j+1}
\end{aligned}
$$
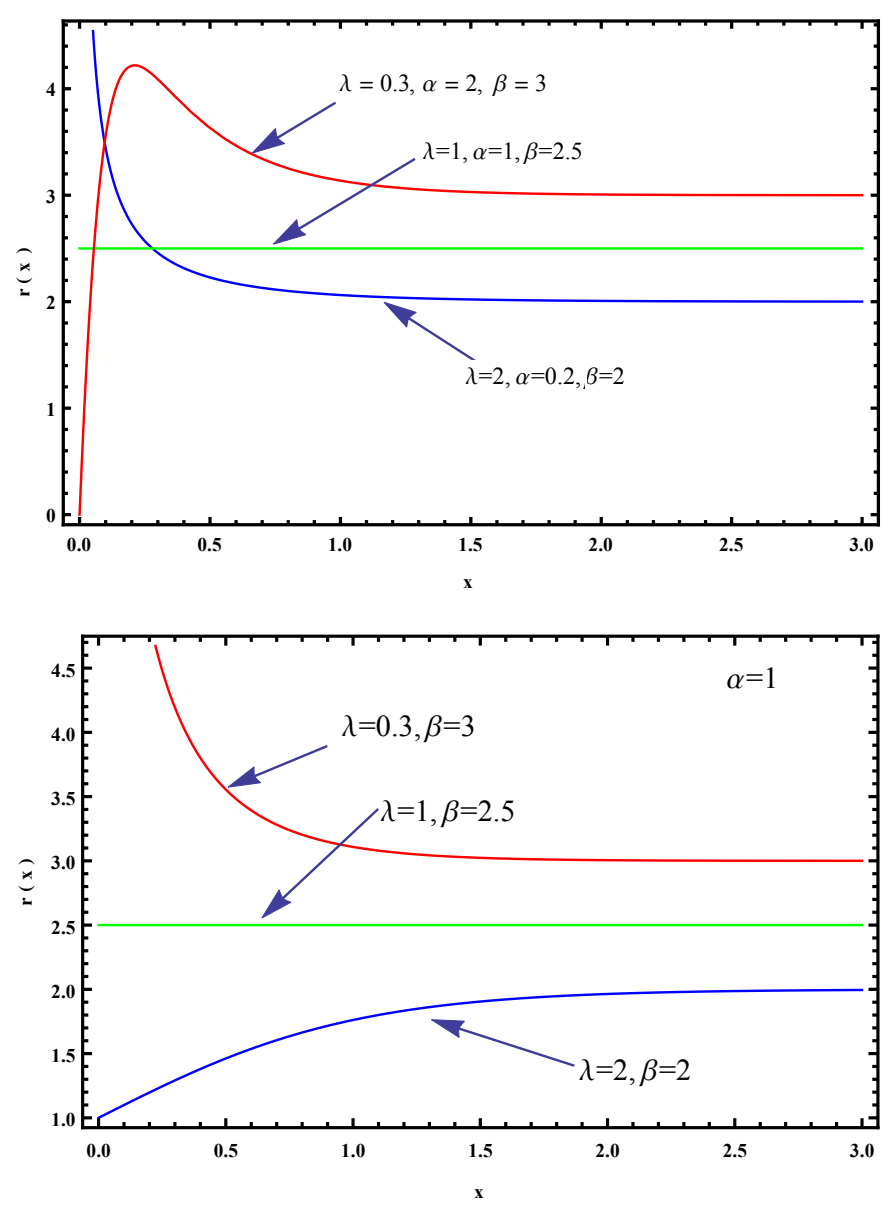

Fig. 2: The $h r f$ of the $M O E E E$ distribution for selected values of the parameters $\alpha, \beta$ and $\lambda$.

\section{MAXIMUM LIKELIHOOD ESTIMATION}

Suppose that $X_{1}, X_{2}, \ldots, X_{n}$ is a random sample of size $n$ from the $M O E E E$ distribution then the likelihood function is given by,

$L_{n}(\alpha, \beta, \lambda)=\prod_{i=1}^{n} g\left(x_{i}, \alpha, \beta, \lambda\right)=\prod_{i=1}^{n} \frac{\alpha \beta \lambda\left(1-e^{-\beta x_{i}}\right)^{\alpha-1} e^{-\beta x_{i}}}{\left(\bar{\lambda}\left(1-e^{-\beta x_{i}}\right)^{\alpha}+\lambda\right)^{2}}$

and the log-likelihood function is

$$
\begin{aligned}
l_{n}= & \sum_{i=1}^{n}(\alpha-1)\left(\log \left(1-e^{-\beta x_{i}}\right)\right)-\beta x_{i}-2 \log \left(\left(\bar{\lambda}\left(1-e^{-\beta x_{i}}\right)^{\alpha}+\lambda\right)\right) \\
& +n \log (\alpha \beta \lambda) .
\end{aligned}
$$

The Maximum Likelihood Estimation (mle ) of $\alpha, \beta$ and $\lambda$ are obtained as the solution of

$$
\frac{\partial l}{\partial \alpha}=0, \frac{\partial l}{\partial \beta}=0, \frac{\partial l}{\partial \lambda}=0 .
$$

Testing the add parameter $\lambda$ in $M O E E E$ distribution : To test the null hypothesis $H_{0}: \lambda=1$ ( $E E$ distribution ), we use the likelihood ratio test (lrt). Under $H_{0}$ the $l r t$ statistic is

$$
X_{L}=2\left[l_{n}(\hat{\alpha}, \hat{\beta}, \hat{\lambda})-l_{n}(\hat{\alpha}, \hat{\beta}, 1)\right]
$$


which has an asymptotic chi-square distribution with 1 degree of freedom. $H_{0}$ is rejected at a significance level of $\alpha$ if $X_{L}>\chi_{(1, \alpha)}^{2}$. In addition, for model selection, we use the Akiake Information Criterion (AIC) and the Bayesian Information Criterion (BIC) defined as:

$$
\begin{gathered}
A I C=\text { loglikelihood }-2 k \\
B I C=\text { loglikelihood }-\frac{k}{2} \log (n)
\end{gathered}
$$

where $k$ is the number of parameters in the model and $n$ is the sample size. For more details about the AIC and BIC, see Akiake [3] and Schwarz et al [17], respectively. The model with higher AIC (BIC) is the one that better fits the data.

\section{APPLICATION}

In this section, we fit the $M O E E E$ distribution to a real data set. This data set is an uncensored data set from Nichols and Padgett [14]consisting of 100 observations on breaking stress of carbon fibers (in Gba) given in table 1

Table 1. : 100 observations on breaking stress of carbon fibers (in Gba)

\begin{tabular}{ccccccccccccccc}
\hline 3.7 & 2.74 & 2.73 & 2.5 & 3.6 & 3.11 & 3.27 & 2.87 & 1.47 & 3.11 & 4.42 & 2.41 & 3.19 & 3.22 & 1.69 \\
3.28 & 3.09 & 1.87 & 3.15 & 4.9 & 3.75 & 2.43 & 2.95 & 2.97 & 3.39 & 2.96 & 2.53 & 2.67 & 2.93 & 3.22 \\
3.15 & 2.35 & 2.55 & 2.59 & 2.38 & 2.81 & 4.2 & 3.33 & 2.55 & 3.39 & 3.31 & 3.31 & 2.85 & 2.56 & 3.56 \\
2.81 & 2.77 & 2.17 & 2.83 & 1.92 & 1.41 & 3.68 & 2.97 & 1.36 & 0.98 & 2.76 & 4.91 & 3.68 & 1.84 & 1.59 \\
2.17 & 1.17 & 5.08 & 2.48 & 1.18 & 3.19 & 1.57 & 0.81 & 5.56 & 1.73 & 1.59 & 2 & 1.22 & 1.12 & 1.71 \\
1.84 & 3.65 & 2.05 & 0.39 & 3.68 & 2.48 & 0.85 & 1.61 & 2.79 & 4.7 & 3.51 & 2.17 & 1.69 & 1.25 & 4.38 \\
2.03 & 1.8 & 1.57 & 1.08 & 2.03 & 1.61 & 2.12 & 1.89 & 2.88 & 2.82 & & & & & \\
\hline
\end{tabular}

The following table gives a comparison between the mles, Loglikelihood, $A I C$ and $B I C$ for the fitted $M O E E E$ and $E E$ distributions to the observations on breaking stress of carbon fibers.

Table 2. : a comparison between the mles, Log-likelihood, $A I C$ and $B I C$ for the fitted $M O E E E$ and $E E$ distributions

\begin{tabular}{|c|c|c|c|c|c|}
\hline Model & Parameters & mle & Log-likelihood & AIC & BIC \\
\hline \multirow{2}{*}{$M O E E E$} & $\alpha$ & 4.42 & & & \\
& $\beta$ & 1.58 & -141.279 & -147.279 & -148.186 \\
\hline \multirow{2}{*}{$E E$} & $\alpha$ & 7.66 & & & \\
& $\beta$ & 1.01 & -146.182 & -150.182 & -150.787 \\
\hline
\end{tabular}

The results of table 2 show that the fitted MOEEE distribution should be selected based on either the $B I C$ or $A I C$ procedure for the given data under $H_{0}, l_{n}(\hat{\alpha}, \hat{\beta}, 1)=-146.182$, thus

$X_{L}=2[-141.279-(-146.182)]=9.806>\chi_{(1,0.05)}^{2}=3.84$,

therefore, we cannot accept the null hypothesis, i.e. the lrt rejects the assumption that the $E E$ model is suitable for the given data. Let $\mathrm{n}$ be the total number of breaking stress of carbon fibers whose survival times, uncensored data, are available. Retable the $\mathrm{n}$ survival times in order of increasing magnitude such that $t_{1} \leq t_{2} \leq$ $\ldots \leq t_{n}$. The Kaplan-Meier estimator $(K M E)$ [9], also known as the product limit estimator, of a $s f$ is defined as:

$$
\bar{G}_{n}(t)=\prod_{t: t_{i} \leq t} 1-\frac{\delta(i)}{n-i+1} \quad, \quad t>0
$$

Figure 3 show the $p-p$ plot of the $E E$ versus the fitted $M O E E E$ $s f$ for the given data. Visually, the depicted points for fitted
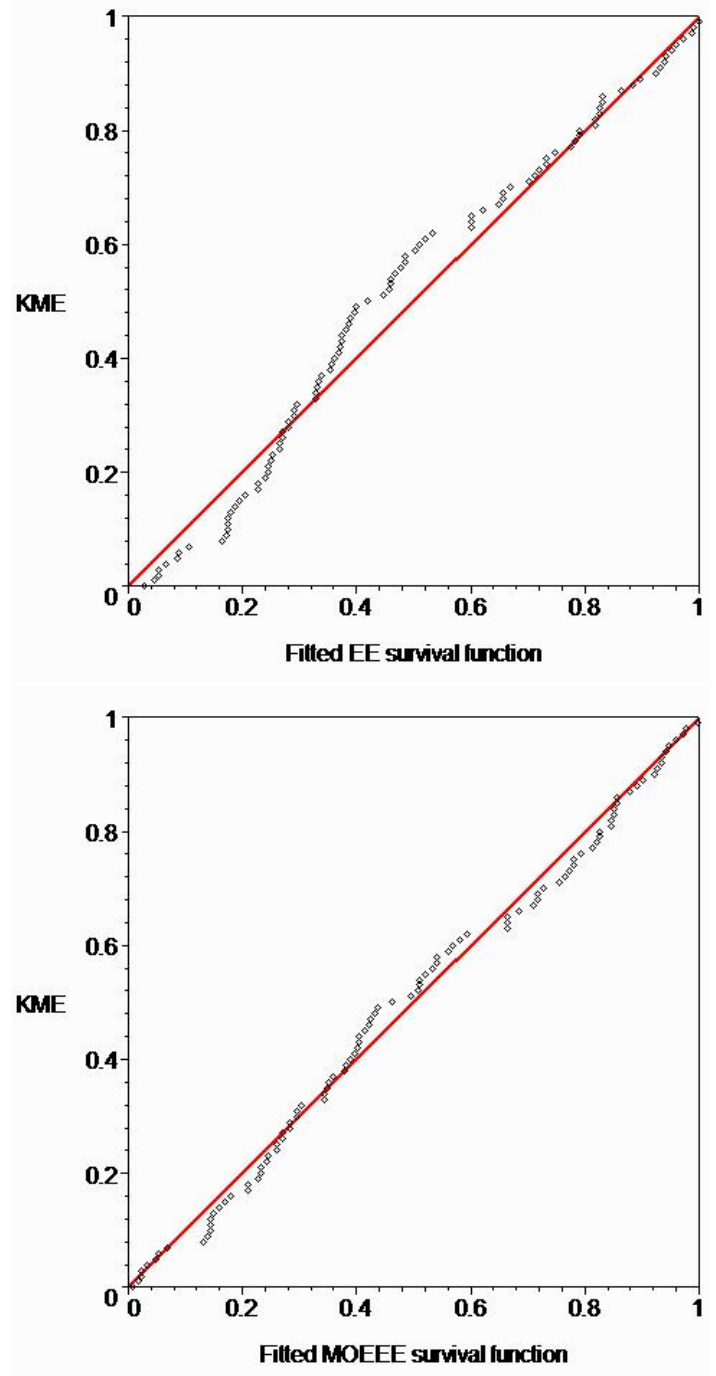

Fig. 3: The $p-p$ plot of the $E E$ versus the fitted MOEEE $s f$ for the given data.

$M O E E E$ survival function are very near the $45^{\circ}$ line, indicating very good fit as compared with the fitted $E E$ survival function. The estimated $h r f r(x)$ of the MOEEE distribution with the estimated parameters $\widehat{\alpha}=4.42, \widehat{\beta}=1.58$ and $\widehat{\lambda}=12.66$, and since $\alpha=4.42>1$ then, by theorem 4 the estimated $r(x)$ is increasing as shown in figure 4

\section{CONCLUSION AND FUTURE WORKS}

The generalization of $E E$ distribution is proposed. The new model, named Marshall-Olkin extend exponentiated exponential (MOEEE) distribution, includes $M O E E$, the $E E$ and the exponential distributions as sub-models. Some reliability properties of the distribution are discussed. The proposed model parameters are estimated by the maximum likelihood method. A real survival data set is analyzed and the results showed that the proposed model was flexible and appropriate. 


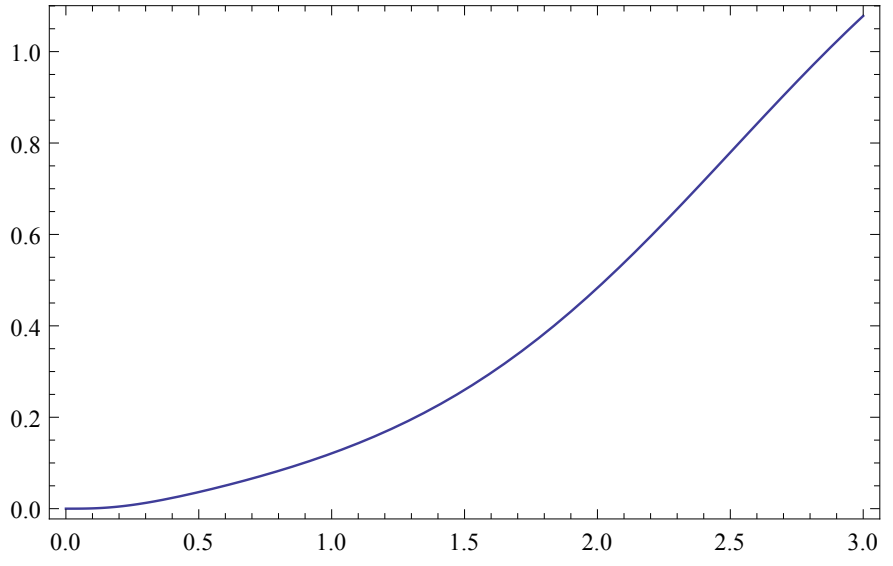

Fig. 4: The estimated $h r f$ of $M O E E E$ distribution to the observations on breaking stress of carbon fibers

\section{ACKNOWLEDGEMENTS}

The authors would like to thank the Editor-in-Chief, the Associate Editor, and the referee for their careful reading and comments which greatly improved the paper.

\section{REFERENCES}

[1] Alaa H Abdel-Hamid and Essam K AL-Hussaini. Estimation in step-stress accelerated life tests for the exponentiated exponential distribution with type-I censoring. Computational Statistics \& Data Analysis, 53(4):1328-1338, 2009.

[2] J. C. Ahuja and Stanley W. Nash. The generalized gompertzverhulst family of distributions. Sankhya : The Indian Journal of Statistics, 29(2):144-156, 061967.

[3] Hirotugu Akaike. Fitting autoregressive models for prediction. Annals of the institute of Statistical Mathematics, 21(1):243-247, 1969.

[4] Muhammad Aslam, Debasis Kundu, and Munir Ahmad. Time truncated acceptance sampling plans for generalized exponential distribution. Journal of Applied Statistics, 37(4):555566, 2010.

[5] Franco Biondi, Tomasz J Kozubowski, Anna K Panorska, and Laurel Saito. A new stochastic model of episode peak and duration for eco-hydro-climatic applications. ecological modelling, 211(3):383-395, 2008.

[6] Rameshwar D Gupta and Debasis Kundu. Generalized exponential distributions. Australian \& New Zealand Journal of Statistics, 41(2):173-188, 1999.

[7] Rameshwar D Gupta and Debasis Kundu. Generalized exponential distribution: Existing results and some recent developments. Journal of Statistical Planning and Inference, 137(11):3537-3547, 2007.

[8] Nandini Kannan, Debasis Kundu, P Nair, and RC Tripathi. The generalized exponential cure rate model with covariates. Journal of Applied Statistics, 37(10):1625-1636, 2010.

[9] Paul Kaplan, E. L.; Meier. Nonparametric estimation from incomplete observations. American Statistical Association, 53(282):457-481, 061958.

[10] Debasis Kundu and Biswabrata Pradhan. Bayesian inference and life testing plans for generalized exponential distribution.
Science in China Series A: Mathematics, 52(6):1373-1388, 2009.

[11] Mohamed T Madi and Mohammad Z Raqab. Bayesian prediction of rainfall records using the generalized exponential distribution. Environmetrics, 18(5):541-549, 2007.

[12] A. Marshall and I. Olkin. A new method for adding a parameter to a family of distributions with application to the exponential and weibull families. Biometrika, 84(3):641-652, 1997.

[13] Saralees Nadarajah and Samuel Kotz. The exponentiated type distributions. Acta Applicandae Mathematica, 92(2):97-111, 2006.

[14] Michele D Nichols and WJ Padgett. A bootstrap control chart for weibull percentiles. Quality and reliability engineering international, 22(2):141-151, 2006.

[15] TA Raja and AH Mir. On extension of some exponentiated distributions with application. Int J Contemp Math Sci, 6:393-400, 2011.

[16] Mohammad Z. Raqab. Inferences for generalized exponential distribution based on record statistics. Journal of Statistical Planning and Inference, 104(2):339-350, 2002.

[17] Gideon Schwarz et al. Estimating the dimension of a model. The annals of statistics, 6(2):461-464, 1978.

[18] DT Shirke, RR Kumbhar, and D Kundu. Tolerance intervals for exponentiated scale family of distributions. Journal of Applied Statistics, 32(10):1067-1074, 2005.

[19] Sanjay Kumar Singh, Umesh Singh, and Abhimanyu Singh Yadav. Parameter estimation in marshall-olkin exponential distribution under type-i hybrid censoring scheme. Journal of Statistics Applications and Probability, 3(2):117-127, 2014.

[20] G. S. Watson and W. T. Wells. On the possibility of improving the mean useful life of items by eliminating those with short lives. Technometrics, 3:281-298, 1961.

[21] MP Yeates, BJ Tolkamp, DJ Allcroft, and I Kyriazakis. The use of mixed distribution models to determine bout criteria for analysis of animal behaviour. Journal of Theoretical Biology, 213(3):413-425, 2001.

[22] Gang Zheng. On the fisher information matrix in type ii censored data from the exponentiated exponential family. Biometrical Journal, 44(3):353-357, 2002. 